\title{
Anharmonic Raman intensities of overtones, combination and difference bands
}

Cite as: J. Chem. Phys. 77, 23 (1982); https://doi.org/10.1063/1.443647

Published Online: 31 August 1998

S. Montero

\section{ARTICLES YOU MAY BE INTERESTED IN}

Infrared spectroscopy of overtones and combination bands

The Journal of Chemical Physics 109, 8641 (1998); https://doi.org/10.1063/1.477531

A second-order perturbation theory route to vibrational averages and transition properties of molecules: General formulation and application to infrared and vibrational circular dichroism spectroscopies

The Journal of Chemical Physics 136, 124108 (2012); https://doi.org/10.1063/1.3695210

First and second order Raman scattering spectroscopy of nonpolar a-plane GaN Journal of Applied Physics 101, 103533 (2007); https://doi.org/10.1063/1.2735402

\section{Lock-in Amplifiers up to $600 \mathrm{MHz}$}

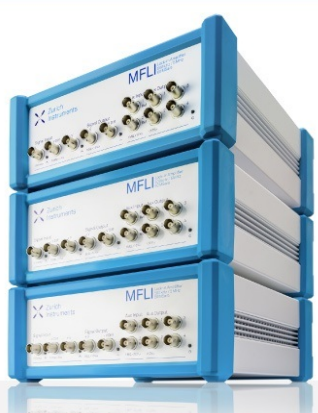




\title{
Anharmonic Raman intensities of overtone, combination and difference bands ${ }^{\text {a) }}$
}

\author{
S. Montero \\ Instituto de Estructura de la Materia, C.S.I.C., Serrano 119, Madrid 6, Spain \\ (Received 12 November 1980; accepted 17 July 1981)
}

The influence of anharmonicity up to the cubic force constants on the Raman tensors of binary overtone, combination, and difference bands is investigated. Formulas for the absolute differential Raman scattering cross sections of these bands are reported for all possible cases of degeneracy as a function of the invariants of the anharmonic Raman tensors. The evaluation of anharmonic Raman tensors as a function of Hoy, Mills, and Strey's $\mathcal{L}$ tensor, cubic force constants, and bond polarizability parameters is illustrated for the tetrahedral $\mathrm{AB}_{4}$ molecule.

\section{INTRODUCTION}

Harmonic approximation formulas for the absolute differential Raman scattering cross sections of binary overtone, combination, and difference bands have been recently reported. ${ }^{1,2}$ Previous works considering the influence of anharmonicity on the dipole vibrational transition moments ${ }^{3-6}$ have shown that, in general, anharmonicity may introduce dramatic perturbations with respect to the values predicted by the harmonic model. Hence, it may also be necessary to take into account anharmonicity in order to go further in the interpretation of the Raman intensities of overtone, combination, and difference bands.

In the present work the anharmonic formulation of the Raman intensities of binary overtone, combination, and difference bands is reported. As an example, detailed expressions for the anharmonic Raman tensors of the tetrahedral $\mathrm{AB}_{4}$ molecule are given as a function of the cubic force constants and of the polarizability parameters (eop's) of the AB bond.

\section{THEORETICAL BACKGROUND}

According to Placzek's theory of the Raman effect, ${ }^{7}$ the absolute differential Raman scattering cross section for a Stokes band related to the transitions between the vibrational levels $i$ and $j$ is given, for a $90^{\circ}$ scatter ing geometry, by the equation

$$
\begin{aligned}
& \left(\frac{\partial \sigma}{\partial \Omega}\right)=(2 \pi)^{4}\left(\nu_{0}-\nu_{i j}\right)^{4} \\
& \times \frac{\sum_{i j} \overline{\left(\left|\left\langle i\left|\alpha_{y y}\right| j\right\rangle\right|^{2}\right.}+\overline{\left.\left.\left\langle i\left|\alpha_{x y}\right| j\right\rangle\right|^{2}\right)} \exp \left(-E_{i} / k T\right)}{\sum_{v} g(v) \exp \left(-E_{v} / k T\right)}
\end{aligned}
$$

where $\nu_{0}$ is the wave number of the monochromatic exciting radiation and $\nu_{1 j}$ the Raman shift of the transitions contributing to the total cross section of the vibrational band; $|i\rangle$ and $|j\rangle$ are the vibrational wave functions of initial and final levels, respectively, $E_{1}$ the energy of the initial level, and $E_{v}$ that of the level with vibrational quantum number $v ; g(v)$ stands for the degeneracy, being $g(v)=v+1$ for doubly degenerate modes and $g(v)=(v+1)(v+2) / 2$ for the triply degenerate ones. $\alpha_{y y}$ and $\alpha_{x y}$ are elements of the molecular polarizability

\footnotetext{
a)Issued as CSIC No. FM90.
}

in the space-fixed reference frame, and the horizontal bar over the transition moments of Eq. (1) refers to the spatial average of the space-fixed frame with respect to that of the randomly oriented molecule; $k$ and $T$ have the usual meaning.

Equation (1) is subject to the limitations of Placzek's theory $y^{7}$ and is only valid for a freely orientable molecule of a sample of noninteracting molecules at thermal equilibrium under the following experimental conditions:

(i) laser beam direction: $X$ axis of the space-fixed frame $(X Y Z)$,

(ii) laser beam polarization: $Y$ axis,

(iii) Raman signal direction: $Z$ axis,

(iv) Raman signal polarization: both components (no analyzer).

In the harmonic approximation, Eq. (1) may be simplified by using the harmonic wave functions $\left|i_{\text {har }}\right\rangle$ and $\left\langle j_{\text {har }}\right\rangle$. Hence, the Raman cross sections of binary overtone, combination, and difference bands may be given in terms of molecular properties, namely, the invariants (trace and anisotropy) of the second derivatives of the molecular polarizability with respect to the normal coordinates. 1,2

How to properly introduce the vibrational anharmonicity is the question now. In principle, this could be done from the beginning by expanding the actual anhar monic wave functions of Eq. (1) in the harmonic basis set

$$
\left|i_{\mathrm{anh}}\right\rangle=\sum_{k} a_{i k}\left|k_{\mathrm{har}}\right\rangle, \quad\left|j_{\mathrm{anh}}\right\rangle=\sum_{k} a_{j k}\left|k_{\mathrm{har}}\right\rangle .
$$

However, the complexity of Eq. (1) soon increases rapidly and its simplification in terms of the molecular polarizability invariants is not evident.

A more elegant procedure arises from the use of an appropriate contact transformation $T$ of the components $\alpha_{y y}$ and $\alpha_{x y}$ of the molecular polarizability in the same theoretical line earlier developed for the dipole moment operator. ${ }^{3-6}$ Accordingly, the vibrational transition moments in Eq. (1) may be expressed as

$$
\left\langle i_{\text {anh }}\left|\left(\alpha_{y y}\right)\right| j_{\text {anh }}\right\rangle=\left\langle i_{\text {har }}\left|\left(\alpha_{y y}\right)_{\text {orf }}\right| j_{\text {har }}\right\rangle \text {, }
$$




$$
\left(\alpha_{y y}\right)_{\text {of }}=\mathrm{T}\left(\alpha_{y y}\right) \mathrm{T}^{-1}
$$

is the transformed or "effective" $y y$ component of the molecular polarizability operator. In the following, $\left(\alpha_{y y}\right)_{\text {off }}$ shall be referred to as the "anharmonic" polar izability. The main advantage of this method is the fact that the anharmonic transition moments are expressed in terms of the harmonic wave functions $\left|i_{\text {has }}\right\rangle$ and $\left|j_{\text {har }}\right\rangle$ of only the initial and final unperturbed vibrational states.

The anharmonic polarizability tensor has the form

$$
\begin{aligned}
\alpha_{2 \mathrm{mh}}= & \mathrm{T} \alpha \mathrm{T}^{-1}=\alpha_{0}+\sum_{s}\left(\frac{\partial \alpha}{\partial q^{s}}\right)_{0} q^{s} \\
& +\frac{1}{2 !} \sum_{s s^{\prime}}\left(\frac{\partial^{s} \alpha}{\partial q^{s} \partial q^{s^{s}}}\right)_{0} q^{s} q^{s^{\prime}}+\hbar \sum_{s \leq s^{\prime}} \sum_{m}\left(\frac{\partial \alpha}{\partial q^{m}}\right)_{0}\left[s_{s s^{\prime}}^{m} q^{s} q^{s^{\prime}}\right. \\
& \left.+S^{s s^{\prime} m}\left(1+\delta_{s m}+\delta_{s^{\prime} m}\right) P_{s^{\prime}} P_{s^{\prime}}\right]+\cdots
\end{aligned}
$$

where $q^{s}$ are dimensionless normal coordinates and $P_{s}$ their conjugate momenta. The coefficients $S_{s s^{\prime}}^{m}$ and $S^{s s^{2} m}$ have been given by Amat, Goldsmith, and Nielsen ${ }^{8}$ as a function of cubic force constants and harmonic wave numbers. The anharmonic Raman tensors of binary overtone, combination, or difference bands may be deduced from Eq. (5), their general form being

$$
\begin{aligned}
& \left(\alpha_{l^{*}}^{\prime \prime}\right)_{\mathrm{anh}}=\left(\frac{\partial^{2} \alpha}{\partial Q_{l} \partial Q_{l^{\circ}}}\right)_{\mathrm{anh}}=\left(\frac{\partial^{2} \alpha}{\partial Q_{l} \partial Q_{l^{*}}}\right)_{h} \\
& +\frac{\hbar\left(1+\delta_{l l^{\circ}}\right)}{\sqrt{2} b_{l} b_{l^{\prime}}} \sum_{m} b_{m}\left(\frac{\partial \alpha}{\partial Q_{m}}\right)_{h} \\
& \times\left[S_{l l^{\circ}}^{m} \mp \hbar^{2} S^{l l^{\prime} m}\left(1+\delta_{l m}+\delta_{l^{\prime} m}\right)\right]+\cdots,
\end{aligned}
$$

where the (-) option stands for overtones and combination bands, and the $(+)$ for the difference bands. $Q$ are mass -weighted normal coordinates (dimensions of $\left.M^{1 / 2} L\right)$ and

$$
b_{1}=\left(h / 8 \pi^{2} \omega_{1} c\right)^{1 / 2}
$$

is the zero vibrational amplitude of the normal mode $Q_{t}$. Substitution of functions $S_{l l^{\prime}}^{m}$ and $S^{l l^{\circ} 8}$ in Eq. (6) yields the explicit form of the Raman tensors whose invariants shall be used in Eqs. (14) to (27) of the present work. These tensors are:

(i) binary overtones:

$$
\begin{aligned}
\left(\frac{\partial^{2} \alpha}{\partial Q_{1}^{2}}\right)_{\mathrm{anh}}= & \left(\frac{\partial^{2} \alpha}{\partial Q_{l}^{2}}\right)_{h}+\frac{1}{3 \sqrt{2} b_{l}} \frac{\phi^{\prime \prime \prime}}{\omega_{1}}\left(\frac{\partial \alpha}{\partial Q_{l}}\right)_{h} \\
& +\sum_{m \neq l} \frac{b_{m}}{\sqrt{2} b_{l}^{2}} \frac{\phi^{l \prime m} \omega_{m}}{\left(4 \omega_{1}^{2}-\omega_{m}^{2}\right)}\left(\frac{\partial \alpha}{\partial Q_{m}}\right)_{h},
\end{aligned}
$$

(ii) binary combination bands:

$$
\begin{aligned}
& \left(\frac{\partial^{2} \alpha}{\partial Q_{l} \partial Q_{l^{\circ}}}\right)_{\text {anh }}^{c}=\left(\frac{\partial^{2} \alpha}{\partial Q_{l} \partial Q_{l^{*}}}\right)_{h}+\frac{1}{\sqrt{2 b_{l^{\prime}}}} \frac{\phi^{3 \prime l^{\circ} \omega_{l}}}{\omega_{l^{\circ}}\left(2 \omega_{l}+\omega_{\eta^{\prime}}\right)}\left(\frac{\partial \alpha}{\partial Q_{l}}\right)_{h} \\
& +\frac{1}{\sqrt{2} b_{l}} \frac{\phi^{l^{\prime \prime} l^{*} \omega_{l}} \omega_{l}}{\omega_{l}\left(2 \omega_{l^{*}}+\omega_{l}\right)}\left(\frac{\partial \alpha}{\left.\partial Q_{l}\right)_{h}}\right. \\
& +\sum_{m \neq i, l} \frac{b_{m}}{\sqrt{2} b_{l} b_{l} \cdot} \frac{\phi^{i * m} \omega_{m}}{\left[\left(\omega_{l}+\omega_{l}\right)^{2}-\omega_{m}^{2}\right]}\left(\frac{\partial \alpha}{\partial Q_{m}}\right)_{h} \text {, }
\end{aligned}
$$

$$
\begin{aligned}
& \left(\frac{\partial^{2} \alpha}{\partial Q_{l} \partial Q_{l^{\circ}}}\right)_{\text {anh }}^{D}=\left(\frac{\partial^{2} \alpha}{\partial Q_{l} \partial Q_{l^{\circ}}}\right)_{n}-\frac{1}{\sqrt{2} b_{l^{*}}} \frac{\phi^{\prime \prime \prime \prime} \omega_{l}}{\omega_{l} \cdot\left(2 \omega_{l}-\omega_{l^{\circ}}\right)}\left(\frac{\partial \alpha}{\partial Q_{l}}\right)_{h} \\
& -\frac{1}{\sqrt{2} b_{l}} \frac{\phi^{l^{\prime \prime} l^{\prime} t} \omega_{l}}{\omega_{l}\left(2 \omega_{l}-\omega_{l}\right)}\left(\frac{\partial \alpha}{\left.\partial Q_{l}\right)_{h}}\right)_{h} \\
& +\sum_{m \neq t, t^{*}} \frac{b_{m}}{\sqrt{2} b_{\imath} b_{l},} \frac{\phi^{\prime l^{\prime} m} \omega_{m}}{\left[\left(\omega_{t}-\omega_{l^{\prime}}\right)^{2}-\omega_{m}^{2}\right]}\left(\frac{\partial \alpha}{\partial Q_{m}}\right)_{h} \text {. }
\end{aligned}
$$

Label $h$ in Eqs. (6) and (8)-(10) stands for harmonic Raman tensors. $\phi^{r s t}$ are the cubic force constants in the representation of adimensional normal coordinates. These force constants are defined according to Ref. 9 , and are given in units of $\mathrm{cm}^{-1}$. They are related to the quadratic $\left(f^{f j}\right)$ and cubic $\left(f^{1 / k}\right)$ force constants in curvilinear coordinates by

$\phi^{r s t}=2 \sqrt{2} b_{r} b_{s} b_{t}\left[f^{i t k} L_{i}^{r} L_{j}^{s} L_{k}^{t}+f^{i s}\left(L_{i}^{r s} L_{j}^{t}+L_{i}^{r t} L_{j}^{s}+L_{i}^{s t} L_{j}^{r}\right)\right]$

with $L_{i}^{r}, L_{i}^{r s}$, etc. being elements of the $\mathscr{L}$ tensor of Hoy, Mills, and Strey ${ }^{9}$ in the representation of internal coor dinates.

Once the concept of anharmonic polarizability has been introduced, the transition moments $\left\langle i_{\text {har }}\left|\alpha_{\text {anb }}\right| j_{\text {har }}\right\rangle$ can be calculated for all possible cases of binary overtone, combination, and difference transitions of nondegenerate, doubly and triply degenerate modes. In order to separate the radial and angular parts of the transition moments, doubly degenerate coordinates were defined as

$$
\begin{aligned}
& Q_{+}=\left(Q_{a}+i Q_{b}\right) / \sqrt{2}=(1 / \sqrt{2}) r e^{i \varphi}, \\
& Q_{-}=\left(Q_{a}-i Q_{b}\right) / \sqrt{2}=(1 / \sqrt{2}) r e^{-i \varphi},
\end{aligned}
$$

while the triply degenerate modes were described in terms of spherical harmonics $Y_{l, m}$ :

$$
\begin{aligned}
& Q_{+}=\left(Q_{c}+i Q_{b}\right) / \sqrt{2}=2 i(\pi / 3)^{1 / 2} r Y_{1,1}, \\
& Q_{-}=\left(Q_{a}-i Q_{b}\right) / \sqrt{2}=-2 i(\pi / 3)^{1 / 2} r Y_{1,-1}, \\
& Q_{0}=Q_{c}=-2 i(\pi / 3)^{1 / 2} r Y_{1,0} .
\end{aligned}
$$

The radial part of the transition moments was evaluated with the aid of the tables for radial matrix elements by Shaffer and Krohn, ${ }^{10}$ whereas the transition moments of the spherical harmonics were calculated using its $3-j$ symbol equivalence. ${ }^{11}$

The calculation of transition moments of overtones of doubly and triply degenerate modes has been explained in some detail in a previous work. ${ }^{2}$ The method followed here has been essentially the same.

Still, an important problem in connection with the sum over vibrational levels of Eq. (1) is the final temperature dependence of the scattering cross section of binary overtone, combination, and difference bands. Fortunately, Yao and Overend ${ }^{4}$ have given a thorough explanation of the influence of antharmonicity on the temperature dependence of vibrational intensities. At moderate temperatures, binary overtone, combination, and difference bands of fundamentals higher than 200 $\mathrm{cm}^{-1}$ have very nearly the same temperature dependence as in the harmonic case. Their conclusions shall be accepted in the present work without further comments.

(iii) binary difference bands: 
Finally, it must be emphasized that the contact trans formation method is not valid under a condition of strong resonance. In such a case, an exact treatment should be required. However, the frontier between resonance and nonresonance is artificial and quite diffuse since, for instance, functions of the form $\left(4 \omega_{l}^{2}\right.$ $\left.-\omega_{m}^{2}\right)^{-1}$ approach continuously to the resonant value $\omega_{m}$ $=2 \omega_{n}$, so that, according to Eqs. (8) to $(10)$, a certain degree of resonance is always present. Establishing clear limits of applicability of the contact transformation method should be the subject of future theoretical work.

The albegraic expansion of the above arguments yields a set of equations for the interpretation of absolute differential Raman scattering cross sections of Stokes transitions, namely, (a) first overtone of a nondegenerate fundamental $Q_{i}$ :

$\left(\frac{\partial \sigma}{\partial \Omega}\right)_{2 \nu_{l}}=\frac{(2 \pi)^{4}}{90} \frac{\left(\nu_{0}-2 \nu_{l}\right)^{4}}{B\left(\nu_{l}\right)^{2}} b_{1}^{4}\left[45 \bar{\alpha}^{2}\left(\alpha_{11}^{\prime \prime}\right)+7 \gamma^{2}\left(\alpha_{1 i}^{\prime \prime}\right)\right]$,

with $b_{i}^{2}=h /\left(8 \pi^{2} \nu_{l} c\right)$ the square of the zero vibrational amplitude of the mode $Q_{1}$ and $\alpha_{11}^{\prime \prime}=\left(\partial^{2} \alpha / \theta Q_{i}^{2}\right)_{\text {anh }}$; the factor $B\left(\nu_{1}\right)^{2}=\left[1-\exp \left(-h \nu_{2} c / k T\right)\right]^{2}$ describes the temperature dependence of the first overtone; (b) first overtone of a doubly degenerate fundamental $Q_{p a}, Q_{p b}$ :

$$
\begin{aligned}
\left(\frac{\partial \sigma}{\partial \Omega}\right)_{2 \nu_{p}}= & \frac{(2 \pi)^{4}}{90} \frac{\left(\nu_{0}-2 \nu_{p}\right)^{4}}{B\left(\nu_{p}\right)^{2}} b_{p}^{4} 2\left[45 \bar{\alpha}^{2}\left(\alpha_{p, p-}^{\prime \prime}\right)\right. \\
& \left.+7 \gamma^{2}\left(\alpha_{p+, p-}^{\prime \prime}\right)+45 \bar{\alpha}^{2}\left(\alpha_{p-, p-}^{\prime \prime}\right)+7 \gamma^{2}\left(\alpha_{p-, p}^{\prime \prime}\right)\right],
\end{aligned}
$$

where

$$
\begin{aligned}
& \alpha_{p \phi, p-}^{\prime \prime}=\left(\alpha_{p a_{, p a}}^{\prime \prime}+\alpha_{p b_{,}, p b}^{\prime \prime}\right) / 2, \\
& \alpha_{p, p-p-}^{\prime \prime}=\left(\alpha_{p a_{, p a}}^{\prime \prime}-\alpha_{p b, p b}^{\prime \prime}+2 i \alpha_{p a_{,}, p b}^{\prime \prime}\right) / 2,
\end{aligned}
$$

with

$$
\alpha_{p a, p a}^{\prime \prime}=\left(\partial^{2} \alpha / \delta Q_{p a}^{2}\right)_{a n h}, \quad \alpha_{p b, p b}^{\prime \prime}=\left(\theta^{2} \alpha / \partial Q_{p b}^{2}\right)_{\alpha a h},
$$

and

$$
\alpha_{p a, s b}^{\prime \prime}=\left(\theta^{2} \alpha / \partial Q_{p a} \partial Q_{p b}\right)_{2 m b} ;
$$

(c) first overtone of a triply degenerate fundamental $Q_{a x}, Q_{a y s} Q_{a x}:$

$$
\begin{aligned}
& \left(\frac{\partial \sigma}{\partial \Omega}\right)_{2 \nu_{q}}=\frac{(2 \pi)^{4}\left(\nu_{0}-2 \nu_{Q}\right)^{4}}{90 B\left(\nu_{q}\right)^{2}} b_{q}^{4} 3\left[45 \vec{\alpha}^{2}\left(\alpha_{\alpha_{,} \alpha}^{\prime \prime}\right)\right. \\
& \left.+7 \gamma^{2}\left(\alpha_{\phi x, 4 x}^{\prime \prime}\right)+14 \gamma^{2}\left(\alpha_{\phi x, 4 y}^{\prime \prime}\right)\right],
\end{aligned}
$$

with $\alpha_{\alpha x_{1} \alpha}^{\prime \prime}=\left(\theta^{2} \alpha / \partial Q_{\alpha x}^{2}\right)_{2 a h}$ and $\alpha_{g x, a y}^{\prime \prime}=\left(\partial^{2} \alpha / \partial Q_{\alpha x} \partial Q_{\alpha y}\right)_{a n h}$; Eq. (17) is invariant with respect to cyclic substitutions in the set of indices $(q x, q y, q z)$; (d) combination bands of two fundamentals $Q_{1}, Q_{m}$ (at least one of the fundamentals, say $Q_{m}$, must be nondegenerate):

$$
\begin{aligned}
\left(\frac{\partial \sigma}{\partial \Omega}\right)_{\nu_{l}+\nu_{m}}= & \frac{(2 \pi)^{4}\left(\nu_{0}-\nu_{l}-\nu_{m}\right)^{4}}{45 B\left(\nu_{l}\right) \cdot B\left(\nu_{m}\right)} \\
& \times b_{l}^{2} b_{m}^{2} g_{l}\left[45 \bar{\alpha}^{2}\left(\alpha_{l m}^{\prime \prime}\right)^{c}+7 \gamma^{2}\left(\alpha_{l m}^{\prime \prime}\right)^{c}\right],
\end{aligned}
$$

with $\left(\alpha_{l m}^{\prime \prime}\right)^{c}=\left(\theta^{2} \alpha / \partial Q, \partial Q_{m}\right)_{\mathrm{anh}}^{c}$ and $g_{t}=1,2$, or 3 being the degeneracy of the fundamental $Q_{l} ;(e)$ difference bands of two fundamentals $Q_{l}, Q_{m}$ (at least one of the fundamentals, say, $Q_{m}$, must be nondegenerate):

$$
\begin{aligned}
\left(\frac{\partial \sigma}{\partial \Omega}\right)_{\nu_{l}-\nu_{m}}= & \frac{(2 \pi)^{4}\left(\nu_{0}-\nu_{l}+\nu_{m}\right)^{4}}{45 B\left(\nu_{l}\right) \cdot A\left(\nu_{m}\right)} \\
& \times b_{l}^{2} b_{m}^{2} g_{l}\left[45 \bar{\alpha}^{2}\left(\alpha_{l m}^{\prime \prime}\right)^{D}+7 \gamma^{2}\left(\alpha_{l m}^{\prime \prime}\right)^{D}\right],
\end{aligned}
$$

with $A\left(\nu_{m}\right)=\left[\exp \left(h \nu_{m} c / k T\right)-1\right]$; as shown in Eqs. (9) and $(10)$, the anharmonic Raman tensors $\left(\alpha_{l m}^{\prime \prime}\right)^{D}$ $=\left(\theta^{2} \alpha / \partial Q_{l} \partial Q_{m}\right)_{a n h}^{D}$ of difference bands and $\left(\alpha_{l m}^{\prime \prime}\right)^{c}=\left(\partial^{2} \alpha\right)$ $\left.\partial Q_{1} \otimes Q_{m}\right)_{2 \pi h}^{c}$ of combination bands may be different in spite of involving the same fundamentals $Q_{1}$ and $Q_{m}$ in

\begin{tabular}{|c|c|c|c|c|c|c|c|c|c|c|c|}
\hline $\begin{array}{l}\text { Symmetry } \\
\text { coordinate }\end{array}$ & $\Delta r_{1}$ & $\Delta r_{2}$ & $\Delta r_{3}$ & $\Delta r_{4}$ & $\Delta\left(a \alpha_{12}\right)$ & $\Delta\left(a \alpha_{34}\right)$ & $\Delta\left(a \alpha_{13}\right)$ & $\Delta\left(a \alpha_{23}\right)$ & $\Delta\left(a \alpha_{24}\right)$ & $\Delta\left(a \alpha_{14}\right)$ & $\begin{array}{l}\text { Normalization } \\
\text { factor }\end{array}$ \\
\hline$S_{1}\left(A_{1}\right)$ & 1 & 1 & 1 & 1 & & & & & & & $2^{-1}$ \\
\hline$s_{2 a}(E)$ & & & & & 2 & 2 & -1 & -1 & -1 & -1 & $12^{-1 / 2}$ \\
\hline$S_{2 b}(E)$ & & & & & & & 1 & -1 & 1 & -1 & $2^{-1}$ \\
\hline$S_{3 x}\left(F_{2}\right)$ & 1 & -1 & -1 & 1 & & & & & & & $2^{-1}$ \\
\hline$s_{4 x}\left(F_{2}\right)$ & & & & & & & & 1 & & -1 & $2^{-1 / 2}$ \\
\hline$S_{3 y}\left(F_{2}\right)$ & -1 & 1 & -1 & 1 & & & & & & & $2^{-1}$ \\
\hline$S_{4 y}\left(F_{2}\right)$ & & & & & & & 1 & & -1 & & $2^{-1 / 2}$ \\
\hline$S_{38}\left(F_{2}\right)$ & 1 & 1 & -1 & -1 & & & & & & & $2^{-1}$ \\
\hline$S_{48}\left(F_{2}\right)$ & & & & & -1 & 1 & & & & & $2^{-1 / 2}$ \\
\hline
\end{tabular}
both cases; (f) combination bands of two doubly degenerate fundamentals $Q_{p a}, Q_{p b}$ and $Q_{q a}, Q_{q b}$ :

$$
\begin{aligned}
\left(\frac{\partial \sigma}{\partial \Omega}\right)_{\nu_{p}+\nu_{q}}= & \frac{(2 \pi)^{4}\left(\nu_{0}-\nu_{p}-\nu_{q}\right)^{4}}{45 B\left(\nu_{p}\right) \cdot B\left(\nu_{q}\right)} b_{p}^{2} b_{\alpha}^{2} 2\left[45 \bar{\alpha}^{2}\left(\alpha_{p+, q-}^{\prime \prime}\right)^{c}\right. \\
& \left.+7 \gamma^{2}\left(\alpha_{p+, q-}^{\prime \prime}\right)^{c}+45 \bar{\alpha}^{2}\left(\alpha_{p-, q-}^{\prime \prime}\right)^{c}+7 \gamma^{2}\left(\alpha_{p-, \sigma}^{\prime \prime}\right)^{c}\right],
\end{aligned}
$$

with

$$
\begin{aligned}
& \left(\alpha_{p+, a}^{\prime \prime}\right)^{c}=\left(\alpha_{p a, q a}^{\prime \prime}+\alpha_{p b, a b}^{\prime \prime}\right)^{c} / 2, \\
& \left(\alpha_{p \rightarrow, q-}^{\prime \prime}\right)^{c}=\left(\alpha_{p a, q a}^{\prime \prime}-\alpha_{p b, a b}^{\prime \prime}+2 i \alpha_{p a, a b}^{\prime \prime}\right)^{c} / 2 ;
\end{aligned}
$$

(g) difference bands of two doubly degenerate fundamentals $Q_{p a s} Q_{p b}$ and $Q_{q a}, Q_{q b}$ :

TABLE I. Symmetry coordinates for the tetrahedral $\mathrm{AB}_{4}$ molecule. Geometry: $A(0,0,0), B_{1}(b,-b, b), B_{2}(-b, b, b), B_{3}(-b,-b,-b)$, $B_{4}(b, b,-b) . \quad b=r_{A B} / \sqrt{3}, a=1 \AA$ as the normalization length. 
TABLE II. $\mathscr{L}_{i}^{r s}$ elements of the $\mathfrak{L}$ tensor for the tetrahedral $\mathrm{AB}_{4}$ molecule in the basis set of Table I as a function of the elements of the $L$ matrix of harmonic vibrational eigenvectors. ${ }^{2}$ Nongiven elements are zero by symmetry. $r=$ bond length; $a=1 \AA$.

\begin{tabular}{|c|c|c|c|}
\hline$l$ & $r$ & $s$ & $\mathcal{L}_{i}^{r s}$ \\
\hline $\begin{array}{l}2 a \\
2 b\end{array}$ & $\begin{array}{l}1 \\
1\end{array}$ & $\begin{array}{l}2 a\} \\
2 b\}\end{array}$ & $-L_{11} L_{22} / 2 r$ \\
\hline $\begin{array}{l}4 x \\
4 y \\
4 z\end{array}$ & $\begin{array}{l}1 \\
1 \\
1\end{array}$ & $\left.\begin{array}{l}3 x \\
3 y \\
3 z\end{array}\right\}$ & $-L_{11} L_{43} / 2 r$ \\
\hline $\begin{array}{l}4 x \\
4 y \\
4 z\end{array}$ & $\begin{array}{l}1 \\
1 \\
1\end{array}$ & $\left.\begin{array}{l}4 x \\
4 y \\
4 z\end{array}\right\}$ & $-L_{11} L_{44} / 2 r$ \\
\hline $\begin{array}{l}1 \\
1\end{array}$ & $\begin{array}{l}2 a \\
2 b\end{array}$ & $\left.\begin{array}{l}2 a) \\
2 b\end{array}\right\}$ & $G_{22} r / 6 a^{2}$ \\
\hline $2 a$ & $2 a$ & $2 a$ & $-G_{22} / 4 \sqrt{6} a$ \\
\hline $2 b$ & $2 a$ & $2 b$ & $-\mathcal{L}_{2 a}^{2 a, 2 a}$ \\
\hline $\begin{array}{l}3 x \\
3 y\end{array}$ & $\begin{array}{l}2 a \\
2 a\end{array}$ & $\left.\begin{array}{l}3 x \\
3 y\end{array}\right\}$ & $r L_{22} L_{43} / 4 a^{2} \sqrt{6}$ \\
\hline $3 z$ & $2 a$ & $3 z$ & $-2 \mathcal{L}_{3 x}^{2 a_{x} 3 x}$ \\
\hline $\begin{array}{l}4 x \\
4 y\end{array}$ & $\begin{array}{l}2 a \\
2 a\end{array}$ & $\left.\begin{array}{l}3 x \\
3 y\end{array}\right\}$ & $-L_{22} L_{33} / 2 r \sqrt{6}$ \\
\hline $4 z$ & $2 a$ & $3 z$ & $-2 \mathcal{L}_{4 x}^{2 a, 3 x}$ \\
\hline $\begin{array}{l}3 x \\
3 y\end{array}$ & $\begin{array}{l}2 a \\
2 a\end{array}$ & $\left.\begin{array}{l}4 x \\
4 y\end{array}\right\}$ & $r L_{22} L_{44} / 4 a^{2} \sqrt{6}$ \\
\hline $3 z$ & $2 a$ & $4 z$ & $-2 \mathcal{L}_{3 x}^{2 a, 4 x}$ \\
\hline $\begin{array}{l}4 x \\
4 y\end{array}$ & $\begin{array}{l}2 a \\
2 a\end{array}$ & $\left.\begin{array}{l}4 x \\
4 y\end{array}\right\}$ & $-L_{22} L_{34} / 2 r \sqrt{6}$ \\
\hline $4 z$ & $2 a$ & $4 z$ & $-2 \mathcal{L}_{4 x}^{2 a, 4 x}$ \\
\hline $3 x$ & $2 b$ & $3 x$ & $\sqrt{3} \mathcal{L}_{3 x}^{2 a, 3 x}$ \\
\hline $3 y$ & $2 b$ & $3 y$ & $-\sqrt{3} \mathcal{L}_{3 x}^{2 a, 3 x}$ \\
\hline $4 x$ & $2 b$ & $3 x$ & $\sqrt{3} \mathcal{L}_{4 x}^{2 a, 3 x}$ \\
\hline $4 y$ & $2 b$ & $3 y$ & $-\sqrt{3} \mathcal{L}_{4 x}^{2 a, 3 x}$ \\
\hline $3 x$ & $2 b$ & $4 x$ & $\sqrt{3} \&_{3 x}^{2 a, 4 x}$ \\
\hline $3 y$ & $2 b$ & $4 y$ & $-\sqrt{3} \mathcal{L}_{3 x}^{2 a, 4 x}$ \\
\hline $4 x$ & $2 b$ & $4 x$ & $\sqrt{3} \mathcal{L}_{4 x}^{2 a, 4 x}$ \\
\hline $4 y$ & $2 b$ & $4 y$ & $-\sqrt{3} \mathscr{L}_{4 x}^{2 a, 4 x}$ \\
\hline $\begin{array}{l}1 \\
1 \\
1\end{array}$ & $\begin{array}{l}3 x \\
3 y \\
3 z\end{array}$ & $\left.\begin{array}{l}3 x \\
3 y \\
3 z\end{array}\right\}$ & $r L_{43} L_{43} / 4 a^{2}$ \\
\hline $2 a$ & $3 x$ & $3 x$ & $\left(-3 L_{43} / 2 \sqrt{6}\right)\left(L_{43} / 4 a+L_{33} / r\right)$ \\
\hline $2 b$ & $3 x$ & $3 x$ & $\sqrt{3} \mathfrak{L}_{2 a}^{3 x, 3 x}$ \\
\hline $2 a$ & $3 y$ & $3 y$ & $\mathcal{L}_{2 a}^{3 x, 3 x}$ \\
\hline $2 b$ & $3 y$ & $3 y$ & $-\sqrt{3} \mathfrak{L}_{2 a}^{3 x, 3 x}$ \\
\hline $2 a$ & $3 z$ & $3 z$ & $-2 \mathcal{L}_{2 a}^{3 x, 3 x}$ \\
\hline $\begin{array}{l}3 z \\
3 x \\
3 y\end{array}$ & $\begin{array}{l}3 x \\
3 y \\
3 z\end{array}$ & $\left.\begin{array}{l}3 y \\
3 z \\
3 x\end{array}\right\}$ & $r L_{43} L_{43} / 8 a^{2}$ \\
\hline $\begin{array}{l}4 z \\
4 x \\
4 y\end{array}$ & $\begin{array}{l}3 x \\
3 y \\
3 z\end{array}$ & $\left.\begin{array}{l}3 y \\
3 z \\
3 x\end{array}\right\}$ & $\left(L_{43} / 2\right)\left(3 L_{43} / 4 a-L_{33} / r\right)$ \\
\hline $\begin{array}{l}1 \\
1 \\
1\end{array}$ & $\begin{array}{l}3 x \\
3 y \\
3 z\end{array}$ & $\left.\begin{array}{l}4 x \\
4 y \\
4 z\end{array}\right\}$ & $r L_{43} L_{44} / 4 a^{2}$ \\
\hline
\end{tabular}

TABLE II (Continued)

\begin{tabular}{|c|c|c|c|}
\hline$l$ & $r$ & $s$ & $\mathcal{L}_{t}^{r s}$ \\
\hline $\begin{array}{l}2 a \\
2 a\end{array}$ & $\begin{array}{l}3 x \\
3 y\end{array}$ & $\left.\begin{array}{l}4 x \\
4 y\end{array}\right\}$ & $-(3 / 4 \sqrt{6})\left[L_{43} L_{44} / 2 a+\left(L_{33} L_{44}+L_{43} L_{34}\right) / r\right]$ \\
\hline $2 a$ & $3 z$ & $4 z$ & $-2 \mathcal{L}_{2 a}^{3 x}, 4 x$ \\
\hline $2 b$ & $3 x$ & $4 x$ & $\sqrt{3} 2_{2 a}^{3 x, 4 x}$ \\
\hline $2 b$ & $3 y$ & $4 y$ & $-\sqrt{3} \mathcal{L}_{2 a}^{3 x, 4 x}$ \\
\hline $3 z$ & $3 x$ & $4 y$ & \\
\hline $3 x$ & $3 y$ & $4 z$ & \\
\hline $3 y$ & $3 z$ & $4 x$ & $r L_{43} L_{44} / 8 a^{2}$ \\
\hline $\begin{array}{l}3 z \\
3 x\end{array}$ & $\begin{array}{l}4 x \\
4 y\end{array}$ & $\begin{array}{l}3 y \\
3 z\end{array}$ & \\
\hline $3 y$ & $4 z$ & $3 x)$ & \\
\hline $4 z$ & $3 x$ & $4 y$ & \\
\hline $4 x$ & $3 y$ & $4 z$ & \\
\hline $4 y$ & $3 z$ & $4 x$ & $3 L_{43} L_{44} / 8 a-\left(L_{33} L_{44}+L_{43} L_{34}\right) / 4 r$ \\
\hline $4 z$ & $4 x$ & $3 y$ & \\
\hline $4 x$ & $4 y$ & $3 z$ & \\
\hline $4 y$ & $4 z$ & $3 x$ & \\
\hline
\end{tabular}

aThe elements $\mathscr{L}_{i}^{4 i, 4 j}(i, j=x, y, z)$ are obtained from $2_{l}^{3 i, 3 j}$ substituting $L_{43}$ by $L_{44}$ and $L_{33}$ by $L_{34}$.

$$
\begin{aligned}
\left(\frac{\partial \sigma}{\partial \Omega}\right)_{\nu_{p}-\nu_{q}}= & \frac{(2 \pi)^{4}\left(\nu_{0}-\nu_{p}+\nu_{q}\right)^{4}}{45 B\left(\nu_{p}\right) \cdot A\left(\nu_{q}\right)} b_{p}^{2} b_{q}^{2} 2\left[45 \bar{\alpha}^{2}\left(\alpha_{p+, \sigma}^{\prime \prime}\right)^{D}\right. \\
& \left.+7 \gamma^{2}\left(\alpha_{p+, \alpha^{-}}^{\prime \prime}\right)^{D}+45 \bar{\alpha}^{2}\left(\alpha_{p-, q_{-}}^{\prime \prime}\right)^{D}+7 \gamma^{2}\left(\alpha_{p-, \alpha_{-}}^{\prime \prime}\right)^{D}\right],
\end{aligned}
$$

with $\left(\alpha_{i j}^{\prime \prime}\right)^{D}$ defined as in Eqs. (21); (h) combination bands of two triply degenerate fundamentals $Q_{D x}, Q_{p y}$, $Q_{p s}$ and $Q_{q x}, Q_{q y}, Q_{\alpha x}$ :

$$
\begin{aligned}
\left(\frac{\partial \sigma}{\partial \Omega}\right)_{\nu_{p}+\nu_{\alpha}}= & \frac{(2 \pi)^{4}\left(\nu_{0}-\nu_{p}-\nu_{q}\right)^{4}}{45 B\left(\nu_{p}\right) \cdot B\left(\nu_{q}\right)} b_{p}^{2} b_{q}^{2} 3\left[45 \bar{\alpha}^{2}\left(\alpha_{p x, q x}^{\prime \prime}\right)^{c}\right. \\
& \left.+7 \gamma^{2}\left(\alpha_{p x, q x}^{\prime \prime}\right)^{C}+14 \gamma^{2}\left(\alpha_{p x, q y}^{\prime \prime}\right)^{c}\right] ;
\end{aligned}
$$

this equation is invariant with respect to cyclic substitutions in the set of indices $x, y, z$; (i) difference bands of two triply degenerate fundamentals $Q_{p x}, Q_{p y y}, Q_{p x}$ and $Q_{Q x}, Q_{\alpha y}, Q_{\alpha x}$

$$
\begin{aligned}
\left(\frac{\partial \sigma}{\partial \Omega}\right)_{\nu_{p}-\nu_{q}}= & \frac{(2 \pi)^{4}\left(\nu_{0}-\nu_{p}+\nu_{q}\right)^{4}}{45 B\left(\nu_{p}\right) \cdot A\left(\nu_{q}\right)} b_{p}^{2} \delta_{q}^{2} 3\left[45 \bar{\alpha}^{2}\left(\alpha_{p x_{,}, \alpha x}^{\prime \prime}\right)^{D}\right. \\
& \left.+7 \gamma^{2}\left(\alpha_{p x, q x}^{\prime \prime}\right)^{D}+14 \gamma^{2}\left(\alpha_{p x, q \nu}^{\prime \prime}\right)^{D}\right] ;
\end{aligned}
$$

(j) combination bands of doubly degenerate fundamental $Q_{p a}, Q_{p b}$ with a triply degenerate fundamental $Q_{\alpha x}, Q_{a y,}$ $Q_{\alpha}:$

$$
\begin{aligned}
\left(\frac{\partial \sigma}{\partial \Omega}\right)_{\nu_{p+\nu_{q}}}= & \frac{(2 \pi)^{4}\left(\nu_{0}-\nu_{p}-\nu_{q}\right)^{4}}{45 B\left(\nu_{p}\right) \cdot B\left(\nu_{q}\right)} b_{p}^{2} b_{\alpha}^{2}\left[14 \gamma^{2}\left(\alpha_{p+, q+}^{\prime \prime}\right)^{c}\right. \\
& \left.+14 \gamma^{2}\left(\alpha_{p+, q-}^{\prime \prime}\right)^{c}+14 \gamma^{2}\left(\alpha_{p+, \alpha 0}^{\prime \prime}\right)^{c}\right]
\end{aligned}
$$

with

$$
\begin{aligned}
& \left(\alpha_{p+, a+}^{\prime \prime}\right)^{c}=\left(\alpha_{p a, a x}^{\prime \prime}-\alpha_{p b, a y}^{\prime \prime}-i \alpha_{p a, a y}^{\prime \prime}-i \alpha_{p b, a x}^{\prime \prime}\right)^{c / 2}, \\
& \left(\alpha_{p+, q-}^{\prime \prime}\right)^{c}=\left(\alpha_{p a, q x}^{\prime \prime}+\alpha_{p b, a y}^{\prime \prime}+i \alpha_{p a, a y}^{\prime \prime}-i \alpha_{p b, a x}^{\prime \prime}\right)^{c} / 2, \\
& \left(\alpha_{p+, a 0}^{\prime \prime}\right)^{c}=\left(\alpha_{p a, a z}^{\prime \prime}-i \alpha_{p b, q z}^{\prime \prime}\right)^{c} / \sqrt{2} ;
\end{aligned}
$$

(k) difference bands of a doubly degenerate fundamental $Q_{p a}, Q_{b b}$ with a triply degenerate fundamental $Q_{a x}, Q_{a y}$ $Q_{\infty}$ : 
TABLE III. First and second derivatives of the molecular polarizability of the tetrahedral molecule $\mathrm{AB}_{4}$ with respect to the symmetry coordinates of Table I.

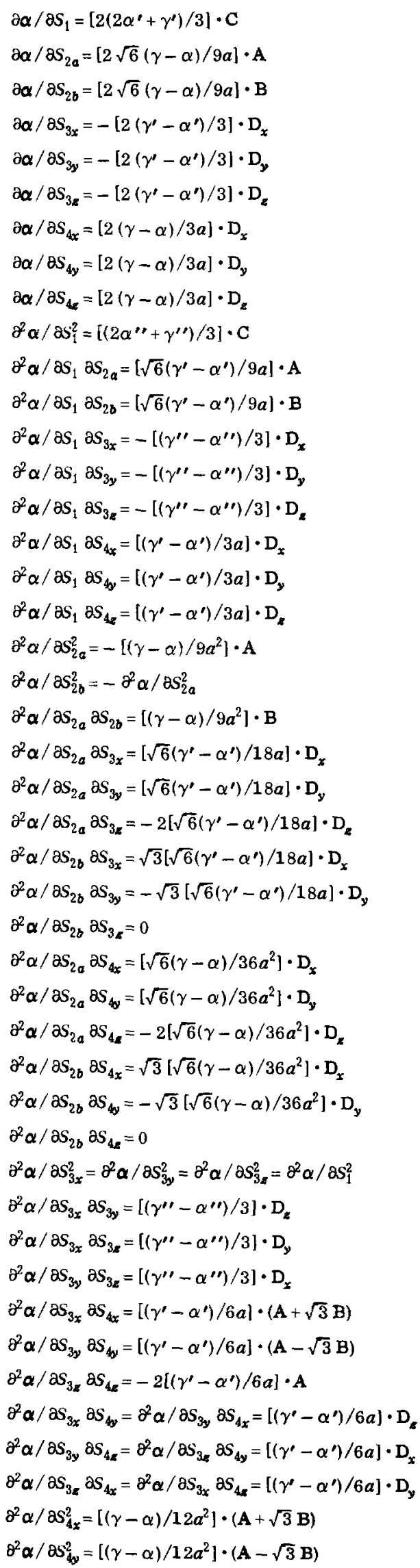

TABLE III (Continued)

$$
\begin{aligned}
& \partial^{2} \alpha / \partial S_{4 x}^{2}=-2\left[(\gamma-\alpha) / 12 a^{2}\right] \cdot \mathrm{A} \\
& \partial^{2} \alpha / \partial S_{4 x} \partial S_{4 y}=\left[7(\gamma-\alpha) / 12 a^{2}\right] \cdot \mathrm{D}_{z} \\
& \partial^{2} \alpha / \partial S_{4 x} \partial S_{4 z}=\left[7(\gamma-\alpha) / 12 a^{2}\right] \cdot \mathrm{D}_{y} \\
& \partial^{2} \alpha / \partial S_{4 y} \partial S_{4 z}=\left[7(\gamma-\alpha) / 12 a^{2}\right] \cdot \mathrm{D}_{x}
\end{aligned}
$$

$$
\begin{array}{lll}
A=\left(\begin{array}{ccc}
1 & 0 & 0 \\
0 & 1 & 0 \\
0 & 0 & -2
\end{array}\right), & \mathbf{B}=\left(\begin{array}{ccc}
\sqrt{3} & 0 & 0 \\
0 & -\sqrt{3} & 0 \\
0 & 0 & 0
\end{array}\right), & \mathrm{C}=\left(\begin{array}{lll}
1 & 0 & 0 \\
0 & 1 & 0 \\
0 & 0 & 1
\end{array}\right), \\
\mathrm{D}_{x}=\left(\begin{array}{lll}
0 & 0 & 0 \\
0 & 0 & 1 \\
0 & 1 & 0
\end{array}\right), & \mathrm{D}_{y}=\left(\begin{array}{lll}
0 & 0 & 1 \\
0 & 0 & 0 \\
1 & 0 & 0
\end{array}\right), & \mathrm{D}_{z}=\left(\begin{array}{lll}
0 & 1 & 0 \\
1 & 0 & 0 \\
0 & 0 & 0
\end{array}\right)
\end{array}
$$

$$
\begin{aligned}
\left(\frac{\partial \sigma}{\partial \Omega}\right)_{\nu_{p}-\nu_{q}}= & \frac{(2 \pi)^{4}\left(\nu_{0}-\nu_{p}+\nu_{q}\right)^{4}}{45 B\left(\nu_{p}\right) \cdot A\left(\nu_{q}\right)} b_{p}^{2} b_{q}^{2}\left[14 \gamma^{2}\left(\alpha_{p+, q+}^{\prime \prime}\right)^{D}\right. \\
& \left.+14 \gamma^{2}\left(\alpha_{p+, q}^{\prime \prime}\right)^{D}+14 \gamma^{2}\left(\alpha_{p+, q 0}^{\prime \prime}\right)^{D}\right],
\end{aligned}
$$

with $\left(\alpha_{i j}^{\prime \prime}\right)^{D}$ defined as in Eqs. (26).

\section{RAMAN TENSORS OF THE TETRAHEDRAL $\mathrm{AB}_{4}$ MOLECULE}

In order to produce the anharmonic Raman tensors according to Eqs. (8) to (10), the harmonic Raman tensors $\left(\partial \alpha / \partial Q_{l}\right)_{h},\left(\partial^{2} \alpha / \partial Q_{l}^{2}\right)_{h}$, and $\left(\partial^{2} \alpha / \partial Q_{l} \partial Q_{l}\right)_{h}$ must first be generated. For this purpose the introduction of the curvilinear symmetry coordinates of Table I has proved to be useful.

The harmonic Raman tensors expressed in terms of curvilinear symmetry coordinates are

$$
\left(\frac{\partial \alpha}{\partial Q_{i}}\right)_{h}=\sum_{l} \mathscr{L}_{i}^{i}\left(\frac{\partial \alpha}{\partial S_{l}}\right)
$$

and

$$
\begin{aligned}
\left(\frac{\partial^{2} \alpha}{\partial Q_{i} \partial Q_{j}}\right)_{h}= & \sum_{i}\left(\frac{\partial \alpha}{\partial S_{l}}\right) \mathcal{L}_{l}^{i j}+\sum_{i}\left(\frac{\partial^{2} \alpha}{\partial S_{l}^{2}}\right) \mathscr{L}_{l}^{i} \mathscr{L}_{l}^{j} \\
& +\sum_{i \neq m}\left(\frac{\partial^{2} \alpha}{\partial S_{l} \partial S_{m}}\right) \mathcal{L}_{l}^{i} \mathcal{L}_{m}^{j},
\end{aligned}
$$

where $\mathcal{L}_{i}^{i}, \mathcal{L}_{i}^{i j}$, etc. are elements of the vibrational $\mathscr{L}$ tensor ${ }^{9}$ in the basis set of Table $I$. Note that the elements $\mathscr{L}_{l}^{i}=L_{l i}$ correspond to the well-known $\mathbf{L}$ matrix of vibrational eigenvectors of the harmonic approximation. The elements $\mathcal{L}_{i}^{i j}$ of the $\mathcal{L}$ tensor are reported in Table II as a function of the elements of the $\mathbf{L}$ matrix.

At this point, two different sources of mechanical anharmonicity may be distinguished. One of them arises from the nonlinear transformation induced by the use of curvilinear coordinates in the description of the normal modes, which are always defined as rectilinear-oscillatory patterns of the atoms. This contribution is apparent in Eq. (11) as a quadratic term $\left(f^{\prime \prime}\right)$ in the cubic force constant $\phi^{\text {rst }}$ and again in Eq. (29) with the term in $\mathcal{L}_{l}^{i j}$. This anharmonicity effect never vanishes, since $\mathcal{L}_{i}^{i j}$ only depends on the harmonic force constants $f^{i j}$ and on the molecular geometry. Indeed, this contribution is more a mathematical artifact rather 
TABLE IV. Harmonic Raman tensors for the $\mathrm{AB}_{4}$ tetrahedral molecule.

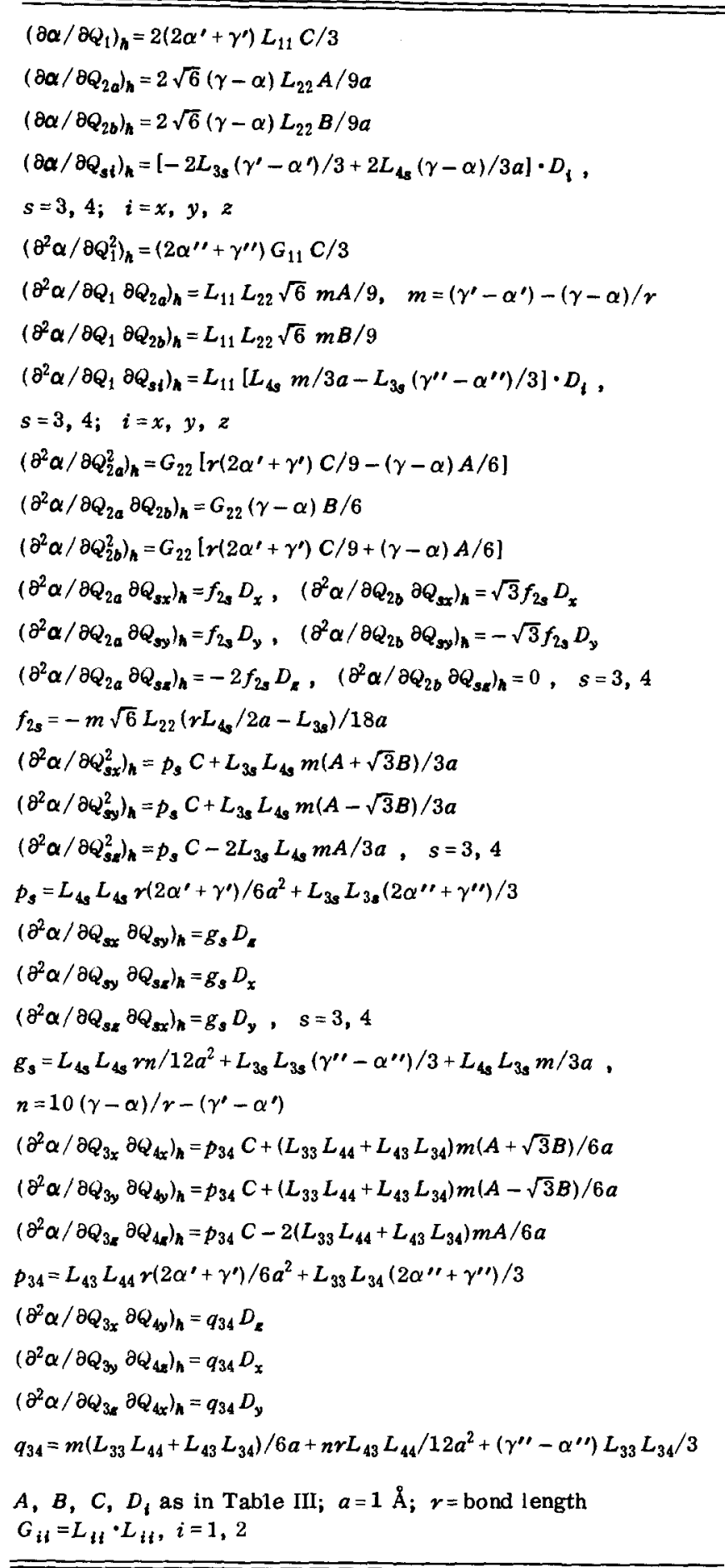

than a physical reality. Thus, the label $h$ in the harmonic Raman tensor $\left(\partial^{2} \alpha / \partial Q_{i} \partial Q_{j}\right)_{h}$ of Eq. (29) can still be justified.

The other source of mechanical anharmonicity is the cubic force constant $f^{i j k}$ in internal coordinates contributing to the cubic force constant $\phi^{\text {rst }}$ in the representation of adimensional normal coordinates [Eq. (11)]. From the physical point of view, $f^{i j k}$ has a simple interpretation, so that the last contribution is closer to the intuitive concept of mechanical anharmonicity than the former.

The first and second derivatives $\left(\partial \alpha / \partial S_{l}\right)$ and $\left(\partial^{2} \alpha / \partial S_{l} \partial S_{m}\right)$, respectively, of the molecular polarizability with respect to the symmetry coordinates [Eqs. (28) and (29)] may be expanded in terms of bond parameters (eop's) according to the methods described elsewhere. ${ }^{1,2}$ The complete set of derivatives necessary to our purpose is given in Table III as a function of the parameters of the polarizability tensor $\alpha_{\mathrm{AB}}$ of the bond AB:

$$
\alpha_{\mathrm{AB}}=\left(\begin{array}{ccc}
\alpha & 0 & 0 \\
0 & \alpha & 0 \\
0 & 0 & \gamma
\end{array}\right)
$$

and its first and second derivatives with respect to the stretching of the bond

$\frac{\partial \alpha_{\mathrm{AB}}}{\partial r_{\mathrm{AB}}}=\left(\begin{array}{ccc}\alpha^{\prime} & 0 & 0 \\ 0 & \alpha^{\prime} & 0 \\ 0 & 0 & \gamma^{\prime}\end{array}\right), \frac{\partial^{2} \alpha_{\mathrm{AB}}}{\partial \gamma_{\mathrm{AB}}^{2}}=\left(\begin{array}{ccc}\alpha^{\prime \prime} & 0 & 0 \\ 0 & \alpha^{\prime \prime} & 0 \\ 0 & 0 & \gamma^{\prime \prime}\end{array}\right)$.

The derivatives of Table III have been calculated in the so-called "zero order approximation" of the bond polarizability model and do not include bond-interaction parameters.

The anharmonic Raman tensors for binary overtone, combination, and difference bands of the $\mathrm{AB}_{4}$ molecule are obtained by substitution of Table IV into Eqs. (8)(10). The symmetry properties of the cubic force constants $\phi^{\text {rst }}$ of the $\mathrm{AB}_{4}$ tetrahedral molecule are reported in Table $\mathrm{V}$. These properties greatly simplify the final expression of the anharmonic Raman tensors. For instance, the intensity of the overtone $2 \nu_{2}$ of $\mathrm{AB}_{4}$ molecules is controlled by the tensors

$$
\begin{aligned}
\left(\frac{\partial^{2} \alpha}{\partial Q_{2 a}^{2}}\right)_{\mathrm{anh}}= & \left(\frac{\partial^{2} \alpha}{\partial Q_{2 a}^{2}}\right)_{h}+\frac{1}{3 \sqrt{2} b_{2}} \frac{\phi^{222}}{\omega_{2}}\left(\frac{\partial \alpha}{\partial Q_{2 a}}\right)_{h}+ \\
& +\frac{b_{1}}{\sqrt{2} b_{2}^{2}} \frac{\phi^{122} \omega_{1}}{\left(4 \omega_{2}^{2}-\omega_{1}^{2}\right)}\left(\frac{\partial \alpha}{\partial Q_{1}}\right)_{h},
\end{aligned}
$$

TABLE V. Symmetry properties of the nonzero cubic force constants $\phi^{\text {rst }}$ of the tetrahedral molecule $\mathrm{AB}_{4}$ in the representation of dimensionless normal coordinates.

$\phi^{122}=\phi^{1,2 a, 2 a}=\phi^{1,2 b, 2 b}$
$\phi^{133}=\phi^{1,3 x, 3 x}=\phi^{1,3 y, 3 y}=\phi^{1,3 z, 3 z}$
$\phi^{134}=\phi^{1,3 x, 4 x}=\phi^{1,3 y, 4 y}=\phi^{1,3 z, 4 z}$
$\phi^{144}=\phi^{1,4 x, 4 x}=\phi^{1,4 y, 4 y}=\phi^{1,4 x, 4}$
$\phi^{222}=\phi^{2 a, 2 a, 2 a}=-\phi^{2 a, 2 b, 2 b}$
$\phi^{233}=\phi^{2 a, 3 x, 3 x}=\phi^{2 a, 3 y, 3 y}=-\frac{1}{2} \phi^{2 a, 3 z, 3 x}=(1 / \sqrt{3}) \phi^{2 b, 3 x, 3 x}=-(1 / \sqrt{3}) \phi^{2 b, 3 y, 3 y}$
$\phi^{234}=\phi^{2 a, 3 x, 4 x}=\phi^{2 a, 3 y, 4 y}=-\frac{1}{2} \phi^{2 a, 3 z, 4 z}=(1 / \sqrt{3}) \phi^{2 b, 3 x, 4 x}=-(1 / \sqrt{3}) \phi^{2 b, 3 y, 4 y}$
$\phi^{244}=\phi^{2 a, 4 x, 4 x}=\phi^{2 a, 4 y, 4 y}=-\frac{1}{2} \phi^{2 a, 4 s, 4 z}=(1 / \sqrt{3}) \phi^{2 b, 4 x, 4 x}=-(1 / \sqrt{3}) \phi^{2 b, 4 y, 4 y}$
$\phi^{334}=\phi^{3 x, 3 y, 4 k}=\phi^{3 x, 4 y, 3 z}=\phi^{4 x, 3 y, 3 z}$
$\phi^{344}=\phi^{3 x, 4 y, 4 z}=\phi^{4 x, 3 y, 4 z}=\phi^{4 x, 4 y, 3 z}$
$\phi^{r s t}$ other than those above given or other than $\phi^{1,1,1}, \phi^{3 x, 3 y, 3 z}$,
and $\phi^{4 x, 4 y, 4 z}$ are zero by symmetry.




$$
\begin{aligned}
&\left(\frac{\partial^{2} \alpha}{\partial Q_{2 b}^{2}}\right)_{2 \mathrm{ab}}=\left(\frac{\partial^{2} \alpha}{\partial Q_{2 b}^{2}}\right)_{h}-\frac{1}{3 \sqrt{2} b_{2}} \frac{\phi^{222}}{\omega_{2}}\left(\frac{\partial \alpha}{\partial Q_{2 a}}\right)_{h} \\
&+\frac{b_{1}}{\sqrt{2} b_{2}^{2}} \frac{\phi^{122} \omega_{1}}{\left(4 \omega_{2}^{2}-\omega_{1}^{2}\right)}\left(\frac{\partial \alpha}{\partial Q_{1}}\right)_{h}, \\
&\left(\frac{\partial^{2} \alpha}{\partial Q_{2 a} \partial Q_{2 b}}\right)_{a \mathrm{abh}}=\left(\frac{\partial^{2} \alpha}{\partial Q_{2 a} \partial Q_{2 b}}\right)_{h}-\frac{1}{3 \sqrt{2} b_{2}} \frac{\phi^{222}}{\omega_{2}}\left(\frac{\partial \alpha}{\partial Q_{2 b}}\right)_{h} .
\end{aligned}
$$

These tensors shall be substituted into Eqs. (16) to generate $\alpha_{2+, 2-}^{\prime \prime}$ and $\alpha_{2-, 2-}^{\prime \prime}$, which are necessary for the interpretation of the Raman intensity of the overtone $2 \nu_{2}$ or, alternatively, to derive information about the cubic force constants $\phi^{122}$ and $\phi^{222}$ from the experimental intensity and depolarization ratio. From Eqs. (32) and (33) it becomes apparent that the isotropic component of $2 \nu_{2}$ will show an appreciable intensity in tetrahedral molecules with $\omega_{1} \sim 2 \omega_{2}$.

\section{FINAL REMARKS}

It has been pointed out before $e^{5}$ that the nine vibrational modes $Q_{1}, Q_{2 a}, Q_{2 b}, Q_{3 x}, Q_{3 y}, Q_{3 x}, Q_{4 x}, Q_{4 y}$, and $Q_{4 x}$ of the tetrahedral $\mathrm{AB}_{4}$ molecule may be regarded as nondegenerate modes when the basis of the Cartesian symmetry coordinates of Table $I$ is used. Thus, the interpretation of the Raman scattering differential cross sections of binary overtone, combination, and difference bands of the $\mathrm{AB}_{4}$ molecule can be accomplished as well with only Eqs. (14), (18), and (19) for the nondegenerate cases. In such a case, the different contributions to each band must be separately calculated. For instance, the intensity of $2 \nu_{3}$ should be interpreted as the sum of intensities of the overtones $2 \nu_{3 x}, 2 \nu_{3 y}$, and $2 v_{3 z}$ and of the combination bands $\nu_{3 x}+\nu_{3 y}, \nu_{3 y}+\nu_{3 k}$, and $\nu_{3 z}+\nu_{3 x}$. This property of the basis set is an additional test for the consistency of the complete set of Eqs. (14) to (27), describing the absolute differential Raman scattering cross sections of all possible binary overtone, combinations, and difference bands of degenerate fundamentals.

Finally, the potential interest of the present approach for obtaining cubic force constants $\phi^{\text {rst }}$ from Raman intensity data should still be emphasized. Due to the resonant terms in the anharmonic Raman tensors [Eqs. $(8)-(10)]$, the Raman intensities of several overtone, combination, and difference bands may be strongly dependent on only one cubic force constant. The magnitude of this cubic force constant might be directly estimated from the intensity ratio of the overtone (or combination, or difference band) with respect to the perturbing fundamental. This would be specially true for perturbing fundamentals belonging to the totally symmetric representation, since the isotropic Raman spectrum $\left(I_{41}-\frac{4}{3} I_{1}\right)$ yields, in these cases, very neat bands without overlapping problems.

\section{ACKNOWLEDGMENTS}

The author is indebted to $\mathrm{C}$. Domingo and $\mathbf{R}$. Escribano for helpful comments on the original manuscript of this paper.

${ }^{1}$ S. Montero, Ind. J. Pure Appl. Phys. 16, 186 (1978) (Raman effect golden jubilee number).

${ }^{2}$ S. Montero, J. Chem. Phys. 72, 2347 (1980).

${ }^{3}$ C. Secroun, A. Barbe, and P. Jouve, J. Mol. Spectrosc. 45, 1 (1973).

${ }^{4}$ S. J. Yao and J. Overend, Spectrochim. Acta Part A 32, 1059 (1976).

5 J. Overend, J. Chem. Phys. 64, 2878 (1976),

${ }^{6} \mathrm{~W}$. G. Golden, C. Marcott, and J. Overend, J. Chem. Phys. 68, 2081 (1978).

${ }^{7} \mathrm{G}$. Placzek, in Handbuch der Radiologie, edited by G. Marx (Akademische Verlagsgesellschaft, Leipzig, 1934), Vol. 6, Part 2.

${ }^{8}$ G. Amat, M. Goldsmith, and H. H. Nielsen, J. Chem. Phys. 27,838 (1957).

${ }^{9}$ A. R. Hoy, I. M. Mills, and G. Strey, Mol. Phys. 24, 1265 (1972).

${ }^{10}$ W. H. Shaffer and B. J. Krohn, J. Mol. Spectrosc. 63,323 (1976),

${ }^{11}$ L. D. Landau and E. M. Lifshitz, Mecánica Cuántica

(Reverte, Barcelona, 1967). 